\title{
EFTEM of Nanoprecipitates: Where Are The Limits?
}

\author{
S. Lozano-Perez, M. L. Jenkins and J. M. Titchmarsh
}

Department of Materials, University of Oxford, Parks Road, OX1 3PH Oxford, UK

Degradation of images in energy-filtered transmission electron microscopy (EFTEM) arises from microscope optics $\left(\mathrm{C}_{\mathrm{s}}\right.$ and $\mathrm{C}_{\mathrm{c}}$ aberrations, diffraction by the objective aperture and delocalization), drift, random noise, sample thickness, background fitting and errors in image cross-correlation We have developed a semi-empirical procedure for estimating the detection and visibility limits of small precipitates as well as the accuracy in measurements of composition and diameter taking these degradation sources into account. The optimisation procedure requires that the signal $(s)$ from a specimen feature of interest be modelled as a function of: number of pre-edge and post-edge energy windows, number of acquisitions of windows, signal intensity, particle size for a fixed sample thickness. We have used for this purpose small spherical $\mathrm{Cu}$ precipitates in thermally aged $\mathrm{Fe}-1 \%$ at.Cu model alloys which were analyzed in a double $\mathrm{C}_{\mathrm{s}}$-corrected JEOL 2200FS (at 200kV) with an $\Omega$-filter. Simulated elemental maps have been produced, artificially degraded and characterized. Once the different contributions are understood it is possible to predict the detection limits and how to optimize acquisition for specific analytical configurations.

Image broadening by the microscope optics has been calculated according to Krivanek [1]. The ideal theoretical image of a particle is then convoluted with a Gaussian function of FWHM equal to the broadening. The specimen is assumed to be stable such that any drift is random in time and direction. An experimental drift distribution for the 2200FS was determined and incorporated into the image simulations [2]. Random noise in the EF images was measured and found to have a Gaussian distribution with a standard deviation $(\sigma)$ approximately three times bigger than expected from an ideal Poisson distribution, due to interaction of the electrons with the CCD [3]. Relative noise was defined as $\sigma(s) / s^{*} 100$, where the background intensity is included in s. Extrapolation of the background under the edge introduces an extra source of noise in the background-subtracted signal, S. An alternative measure of the image quality is the signal-to-noise ratio (SNR), defined as $S / \sigma$. Sample thickness greater than the inelastic scattering mean free path decreases the signal-tobackground ratio (SBR), and thus the SNR, through multiple scattering [4]. Traditionally, two preedge windows are used to extrapolate under the edge. However, increasing the number of background fitting windows can improve the SNR. Fig. 1a shows how a similar reduction in the relative error can be achieved either by using 2 (repeated and summed) acquisitions of 2 pre-edge images, or using 3 pre-edge images, the latter saving the time of one acquisition. Larger numbers of pre-edge windows obviously give greater improvements. Provided the images have enough contrast, sub-pixel accuracy can be achieved in the image cross-correlation and therefore this degradation source has been ignored [5].

Once these effects were evaluated, the following conclusions were reached:

- For a given total data acquisition time, the optimised SNR and detection limits vary with the number of pre-edge windows used. Further improvement can be achieved by also averaging multiple pre-edge images, at the cost of a higher electron exposure (Fig. 1b).

- It is possible to model the relationship between the specific drift and detector noise characteristics of the microscope and the detection limits. 
- For the given experimental conditions, imaging of $1 \mathrm{~nm}$-diameter $\mathrm{Cu}$ precipitates using $\mathrm{Fe}$ or $\mathrm{Cu}$ elemental maps could be achieved by averaging the post-edge image over 50 acquisitions and using multiple pre-edge images (Fig. 2).

\section{References}

1. O. L. Krivanek, M. K. Kundmann et al., Journal of Microscopy, 180, (1995), 277-287.

2. S. Lozano-Perez, J. M. Titchmarsh et al., Ultramicroscopy, (Submitted), (2005),

3. R. R. Meyer, A. I. Kirkland, Microscopy Research and Technique, 49, (2000), 269-280.

4. M. Watanabe, D. B. Williams et al., Micron, 34, (2003), 173-183.

5. B. Schaffer, W. Grogger et al., Ultramicroscopy, 102, (2004), 27-36.
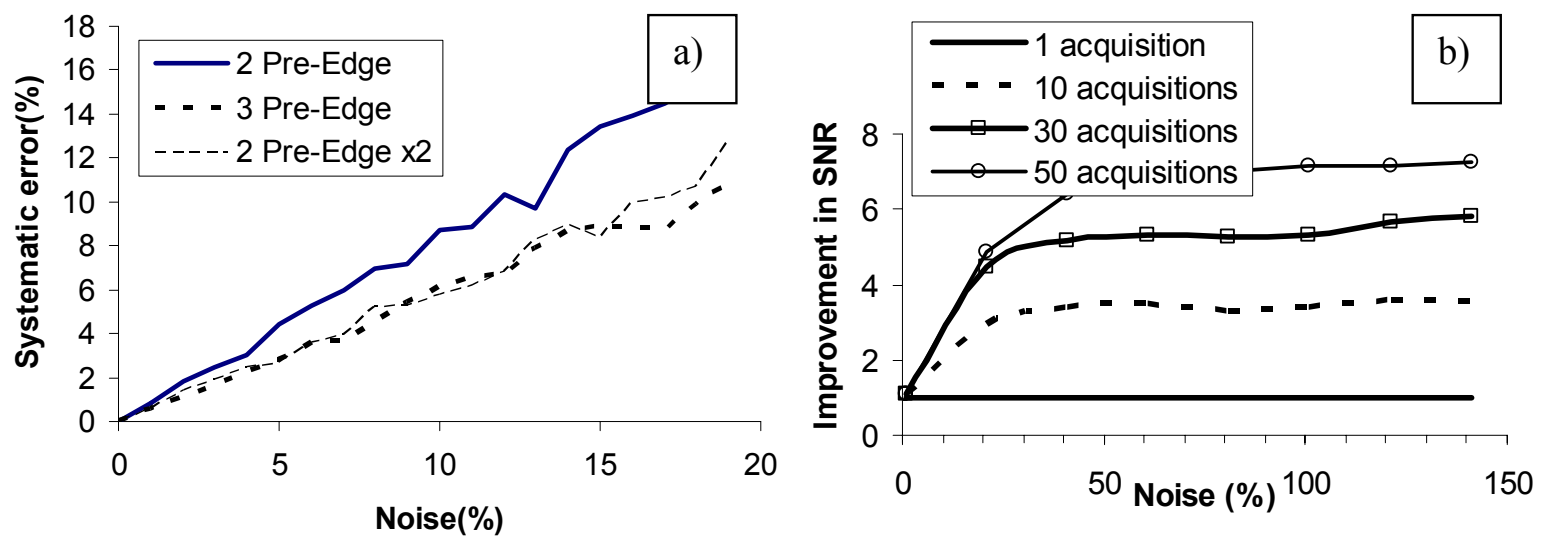

Fig. 1 (a) Systematic error in extrapolated background signal using various edge combinations; (b) Qualitative improvement in the SNR when increasing the number of acquisitions
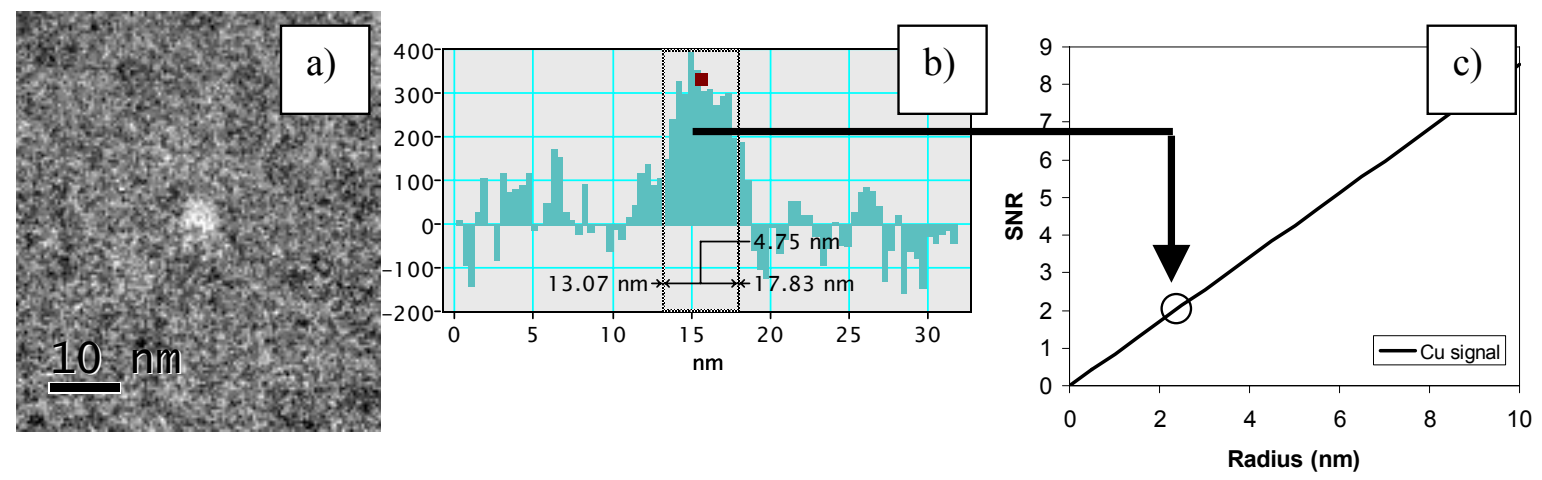

Fig. 2. (a) Cu elemental map using 3-windows and 1 acquisition; (b) Line profile across precipitate; (c) Expected SNR vs. precipitate size for the Cu signal (L23 edge, 40eV slit width, $12 \mathrm{mrad}$ OA aperture, $50 \mathrm{~s}$ acq time, $20 \%$ noise). 